Critical strain region evaluation of self-assembled semiconductor quantum dots

This article has been downloaded from IOPscience. Please scroll down to see the full text article. 2007 Nanotechnology 18475503

(http://iopscience.iop.org/0957-4484/18/47/475503)

The Table of Contents and more related content is available

Download details:

IP Address: 150.214.76.200

The article was downloaded on 07/07/2009 at 12:13

Please note that terms and conditions apply. 


\title{
Critical strain region evaluation of self-assembled semiconductor quantum dots
}

\author{
D L Sales ${ }^{1}$, J Pizarro $^{2}$, P L Galindo ${ }^{2}$, R Garcia ${ }^{1}$, G Trevisi ${ }^{3}$,

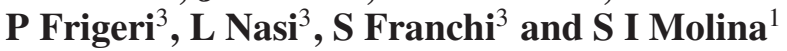 \\ ${ }^{1}$ Departamento de Ciencia de los Materiales e I. M. y Q. I., Universidad de Cádiz, \\ Puerto Real, Cádiz, Spain \\ ${ }^{2}$ Departamento de Lenguajes y Sistemas Informáticos, Universidad de Cádiz, Puerto Real, \\ Cádiz, Spain \\ ${ }^{3}$ CNR-IMEM Institute, Parco delle Scienze 37a, 43100, Parma, Italy
}

E-mail: david.sales@uca.es

Received 23 April 2007, in final form 24 September 2007

Published 19 October 2007

Online at stacks.iop.org/Nano/18/475503

\begin{abstract}
A novel peak finding method to map the strain from high resolution transmission electron micrographs, known as the Peak Pairs method, has been applied to $\operatorname{In}(\mathrm{Ga}) \mathrm{As} / \mathrm{AlGaAs}$ quantum dot (QD) samples, which present stacking faults emerging from the QD edges. Moreover, strain distribution has been simulated by the finite element method applying the elastic theory on a 3D QD model. The agreement existing between determined and simulated strain values reveals that these techniques are consistent enough to qualitatively characterize the strain distribution of nanostructured materials. The correct application of both methods allows the localization of critical strain zones in semiconductor QDs, predicting the nucleation of defects, and being a very useful tool for the design of semiconductor devices.
\end{abstract}

\section{Introduction}

Strain-induced quantum dot (QD) growth using the StranskiKrastanov mode [1] gives the opportunity to approach the ideal zero dimensional system with atom-like properties [2]. GaAs substrates have significant advantages over InP, mostly used in actual devices. They are the larger size of commercially available wafers, with lower fragility and lower cost. In this way, self-assembled $\operatorname{In}(\mathrm{Ga})$ As quantum dots on GaAs substrates are the object of many recent studies because of their promising applications in next generation optoelectronic devices, like infrared photodetectors $[3,4]$, long wavelength lasers [5, 6] and optical memories [7, 8]. One of the most important features of these nanostructures is their strain distribution. It plays a key role in the formation of the QDs and in the determination of their physical and mechanical behaviour, like piezoelectric and optical properties, degeneration and mechanical stability of QDs [9-11]. Furthermore, high strain concentration areas appear during the QD growth, thus making energetically favourable the formation of misfit dislocations when the coverage exceeds a critical thickness $[12,13]$. Typically, these dislocations lead to defect nucleation in the upper confining layer [14]. It has been demonstrated that both the PL intensity and PL decay duration strongly decrease with the formation of these dislocated clusters, which act as non-radiative centres $[15,16]$. Therefore, techniques which give additional information for a better understanding of defect generation in QD structures are relevant.

Strain can be mapped with nanometric resolution by applying image processing techniques to high resolution transmission electron microscopy (HRTEM) images obtained in conditions for which a constant spatial relationship between the intensity maxima of the image and the relative positions of the atomic columns in the specimen exists. The most common algorithms used for strain mapping from HRTEM images are based on peak finding (real space) [17] and geometric phase (Fourier space) [18]. Peak finding methods work in real space, building a two-dimensional reference lattice associated with a non-distorted region of the material, and identifying the local displacements of a grid that is built up from the set of intensity maxima in the HRTEM image. The strain distribution is easily derived from lattice displacements. A new peak finding 


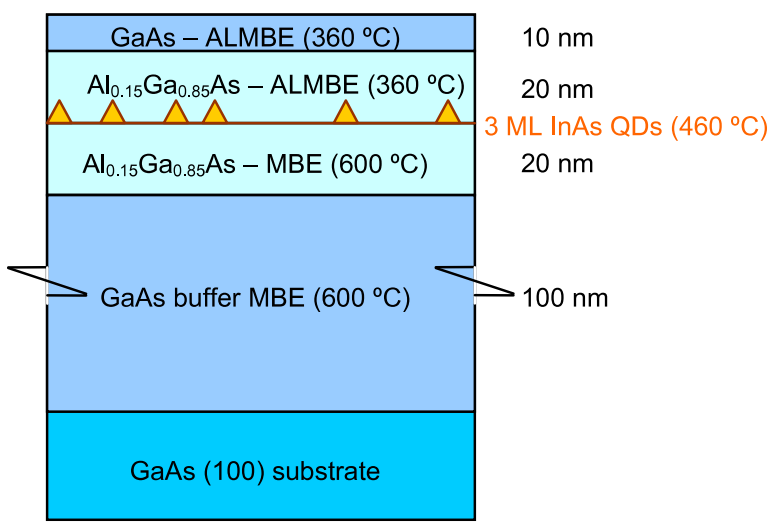

Figure 1. Schematic diagram of InAs/AlGaAs QD structures. Thicknesses and growth temperatures used for each layer are shown. (This figure is in colour only in the electronic version)

method, known as the Peak Pairs method [19], has recently been introduced to map the strain from HRTEM images. This method is based on the detection of pairs of intensity maxima in the affine transformed space, which allow reliable strain analysis even when defects are present in the image.

Several methods have been used to simulate the strain distribution in buried QDs, the finite element method (FEM) being the most extended [20, 10]. Among its advantages we can mention lower computing and time requirements, allowing the use of arbitrary shape structures with anisotropic properties and non-uniform composition.

In the present work, we study the strain distribution of $\mathrm{In}(\mathrm{Ga}) \mathrm{As} / \mathrm{AlGaAs}$ self-assembled semiconductor QDs to contribute to the understanding and prediction of defect generation in order to improve the design of these nanostructures. In this sense, the strain map generated in and around an $\mathrm{In}(\mathrm{Ga}) \mathrm{As}$ QD has been experimentally determined with nanometric resolution from high resolution transmission electron microscopy (HRTEM) images and compared with the strain calculated by the finite element method using the elastic theory.

\section{Experimental set-up}

The studied samples consist of $\operatorname{In}(\mathrm{Ga}) \mathrm{As} / \mathrm{Al}_{0.15} \mathrm{Ga}_{0.85} \mathrm{As}$ self-assembled QDs, grown on GaAs substrates by both conventional molecular beam epitaxy (MBE) [21] and atomic layer MBE (ALMBE) [22, 23]. As shown in figure 1, the structures comprise (i) a GaAs buffer layer and a $20 \mathrm{~nm}$ thick $\mathrm{Al}_{0.15} \mathrm{Ga}_{0.85}$ As lower confining layer (LCL) grown by $\mathrm{MBE}$ and (ii) a 3.0 monolayer (ML) InAs QD layer grown by ALMBE. The ALMBE growth technique consists of an alternated supply of cations and anions to the surface and allows the achievement of a sufficiently high adatom surface mobility even at relatively low growth temperature. In order to reduce the group-III atom interdiffusion and In-segregation effects on QD composition, the upper confining layers (UCLs) and cap layers of the structures in figure 1 are prepared by ALMBE at growth temperatures lower than that used for the QD deposition. Growth temperature and thickness for each layer are shown in figure 1. The insertion of $\mathrm{Al}$ in the $\mathrm{QD}$ confining layer is a well known design strategy which provides, for a constant lattice mismatch, wider band discontinuities to confine carriers in QDs, leading to higher transition energies and emission efficiencies at room temperature [24].

Cross-section electron-transparent specimens were prepared by thinning down to $100 \mu \mathrm{m}$ by mechanical grinding and dimpling down to $20 \mu \mathrm{m}$, followed by ion milling at $4.5 \mathrm{kV}$ to electron transparency. Transmission electron microscopy (TEM) images were taken using a JEOL 2011 microscope, working at $200 \mathrm{kV}$.

\section{Strain mapping determination: the Peak Pairs approach}

The Peak Pairs method is a novel refined method to get strain maps from HRTEM micrographs [19]. The first stage of the procedure is to apply a Wiener filter, in order to reduce the high frequency noise level. Additionally, a Bragg filter is also applied to the Fourier-transformed image to discriminate the non-periodic information. Special attention has been paid to the filtering stage in order to avoid loss of significant information. Local intensity maxima are identified on a pixel basis on the Bragg-filtered image, achieving subpixel resolution by $2 \mathrm{D}$ interpolation followed by function maximization. Two non-collinear vectors are selected as the basis vectors that will be used as references for the strain determination. The reference area has been taken on the same image, but outside deformed regions. Strain is determined from the distortions in the image as compared to the reference (unstrained) vectors.

Figure 2(a) shows an HRTEM image of a single InAs QD with two stacking faults. These defects, which are found at several locations in the sample, appear to be connected with the QD edges. The resultant image after applying the Peak Pairs method is shown in figure 2(b). This image shows the strain $\varepsilon_{[001]}$ along the [001], i.e. the growth, direction. Positive strain values (red coloured zone) correspond to the InAs QD and wetting layer (WL), caused by the distortion as a consequence of the reticular misfit existing between the two materials (InAs/AlGaAs). The most strained region is located in the core of the QD (marked with a square in figure 2(b)) and has an average strain value of $0.058 \pm 0.015$. Additionally, the strain map gives further information on the measured size, shape and location of the studied quantum dot with respect to the contrast phase image, which normally presents dark contrasts that can be wrongly identified as the QD.

A negative strain concentration area (dark blue zone) appears over the quantum dot. Looking at low magnification TEM 002 bright field images (figure 3(b)), dark contrast can also be localized in the upper part of the QDs. This contrast reveals deformation of $\{002\}$ planes.

Stacking faults appear in red in figure 2(b) due to the change in the stacking sequence. These high strain values allow a good visualization of these defects in the strain map, though they do not correspond to real strain but to displacement of lattice planes to both sides of the stacking fault plane.

\section{Strain mapping calculation: FEM model}

Finite element method (FEM) modelling was carried out in order to simulate the stress and strain in a three-dimensional 


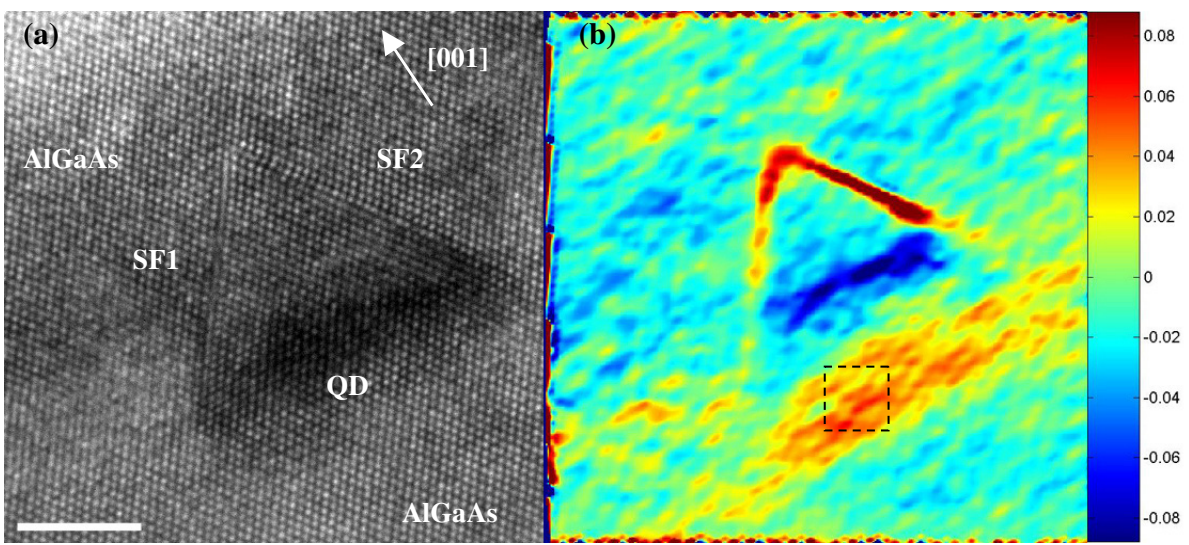

Figure 2. (a) Cross-sectional high resolution transmission electron micrograph of a quantum dot with two stacking faults $\left(\mathrm{SF}_{1}\right.$ and $\left.\mathrm{SF}_{2}\right)$. (b) Strain along [001] mapped as a result of the Peak Pairs method application. The maximum strain region is marked. Scale bar 5 nm.
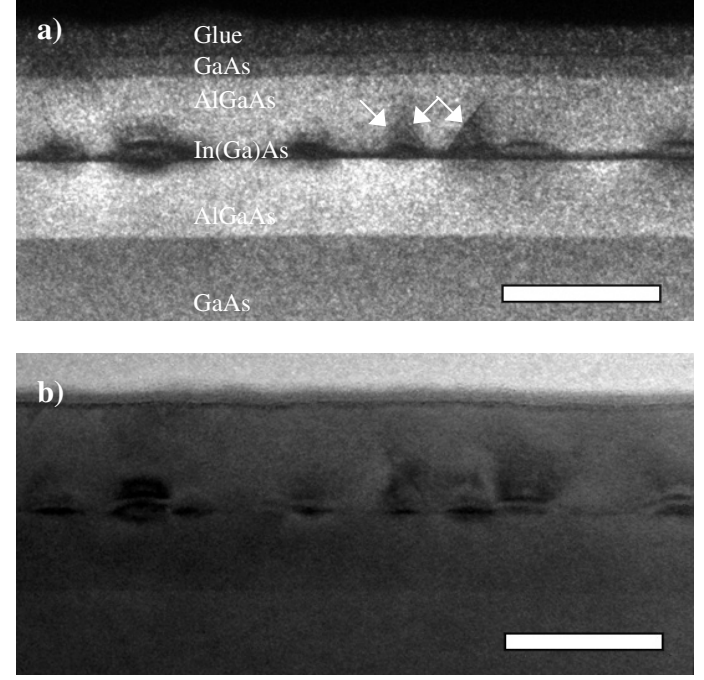

Figure 3. Cross-sectional (002) dark field (a) and bright field (b) TEM images. White arrows point to stacking faults. Scale bar $50 \mathrm{~nm}$.

QD heterostructure. The model was defined taking into account shape, composition, boundary conditions and elastic parameters of the materials.

\subsection{Shape}

The shape of the dot structure has been modelled on the basis of two beam conditions, (002) dark field (figure 3(a)) and HRTEM images. Then, a 3D truncated cone shape is defined, having 11 and $5 \mathrm{~nm}$ for major and minor radii respectively, and a height of $6 \mathrm{~nm}$ starting from the lower $\mathrm{AlGaAs} / \mathrm{In}(\mathrm{Ga}) \mathrm{As}$ interface. The wetting layer is $3 \mathrm{~nm}$ high. The LCL thickness was chosen to be $20 \mathrm{~nm}$, a value large enough that a further increase does not influence the results.

\subsection{Composition: the In segregation effect}

It is well known that the In segregation process takes place during the epitaxial deposition of the overlayer. This phenomenon consists in a thermally activated process, where the In atoms migrate towards the growth front. As a

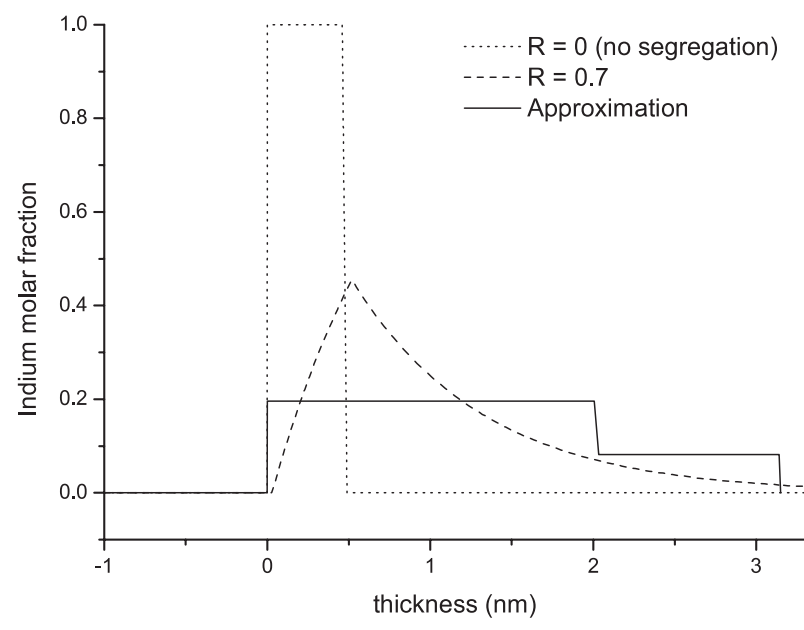

Figure 4. Indium concentration profile for the wetting layer without segregation (dotted line), with a segregation parameter $R=0.7$ (dashed line) and the selected approximation for the FEM model (solid line).

consequence, the thickness and composition of WL and QDs are different from the nominal ones. In particular, experimental cross-section TEM analyses of capped QDs provide values of the WL thickness larger than the critical one, corresponding to the transition from $2 \mathrm{D}$ strained layer to $3 \mathrm{D}$ islands. Experimental evidence and theoretical calculations showed that this critical thickness is about 1.3-1.7 MLs depending on growth conditions [25].

4.2.1. Wetting layer composition. Segregation is definitely present in the studied structure, as the observed thickness for the WL is higher than $3 \mathrm{~nm}$, the critical thickness for the MBE growth of InAs being smaller than $0.52 \mathrm{~nm}(1.7 \mathrm{ML})$. The segregation of In atoms towards the growth front during the deposition of the overlayer makes the WL thickness larger, and the In composition more diluted: the evaluation of the In composition profile in the WL is thus necessary.

In the literature, different approaches have been used to quantify the In segregation effect for the $\operatorname{In}(\mathrm{Ga}) \mathrm{As} / \mathrm{GaAs}$ system grown by MBE, the Muraki model for the segregation [26] 


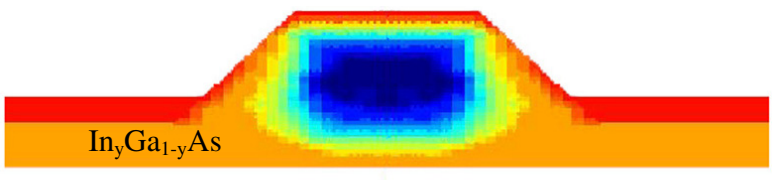

In molar fraction $(y)$

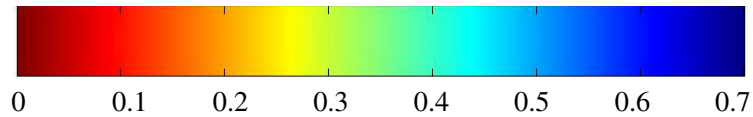

Figure 5. Distribution map for group-III elements of the wetting layer and QD used in the FEM model. The molar fraction of indium $(y)$ is represented following the adjacent colour scale.

being one of the most common $[27,28]$. The dashed line in figure 4 shows the In distribution profile calculated by this model, using a segregation parameter $R=0.7$. This profile shows a good fitting with the WL contrast profile of figure 3(a). For the sake of simplicity, a step-graded In composition profile subtending the same area of the dashed curve was chosen (solid line) to perform the simulations. We also illustrate in figure 4 the In profile for a WL that is not subjected to segregation effects (dotted line).

4.2.2. Quantum dot composition. In the same way, In segregation is expected to occur in the 3D nanostructures, leading to an In distribution that has the maximum value in the core of the QD and that decreases away from it, as shown by several authors [29-31]. Figure 5 shows the simplified distribution assumed in the present work. To get this map, a material balance has been performed, considering the QD average volume, density of QDs in the sample $\left(1.1 \times 10^{11} \mathrm{~cm}^{-2}\right)$, measured with AFM, and the maximum In composition in the QD core, that we set as 0.70. The presence of $\mathrm{Al}$ in the $\mathrm{QD}$ is neglected, as the $\mathrm{Al}$ activation energy for diffusion is higher than that for $\mathrm{Ga}$ [32] and the nominal $\mathrm{Al}$ composition is low $\left(x_{\mathrm{Al}}=0.15\right)$. In any case, aluminium would imply no considerable change in the system strain due to the small difference between AlAs/GaAs lattice parameters.

\subsection{Boundary conditions}

A three-dimensional quantum dot is modelled and symmetries are then incorporated into the calculations to represent the entire sample. The periodicity of the structure is chosen to be $30 \mathrm{~nm}$ and it is taken into account by applying the appropriate boundary conditions: all the nodes of symmetry planes are fixed against displacement in the directions normal to these planes, and all nodes on the bottom of the substrate are fixed against rigid body shift in the $z$-direction.

\subsection{Subdomains and mesh}

Five subdomains have been defined: (1) $\mathrm{Al}_{0.15} \mathrm{Ga}_{0.85} \mathrm{As}$ upper confining layer, (2) $\mathrm{In}_{0.10} \mathrm{Ga}_{0.90}$ As wetting layer zone, (3) $\mathrm{In}_{0.20} \mathrm{Ga}_{0.80}$ As wetting layer zone, (4) non-uniform composition $\mathrm{In}_{x} \mathrm{Ga}_{(1-x)}$ As quantum dot, and (5) $\mathrm{Al}_{0.15} \mathrm{Ga}_{0.85}$ As lower confining layer. The basic mesh composition for finite element calculation has been generated using the Delaunay algorithm [33], that can be applied for all geometry objects. Cells

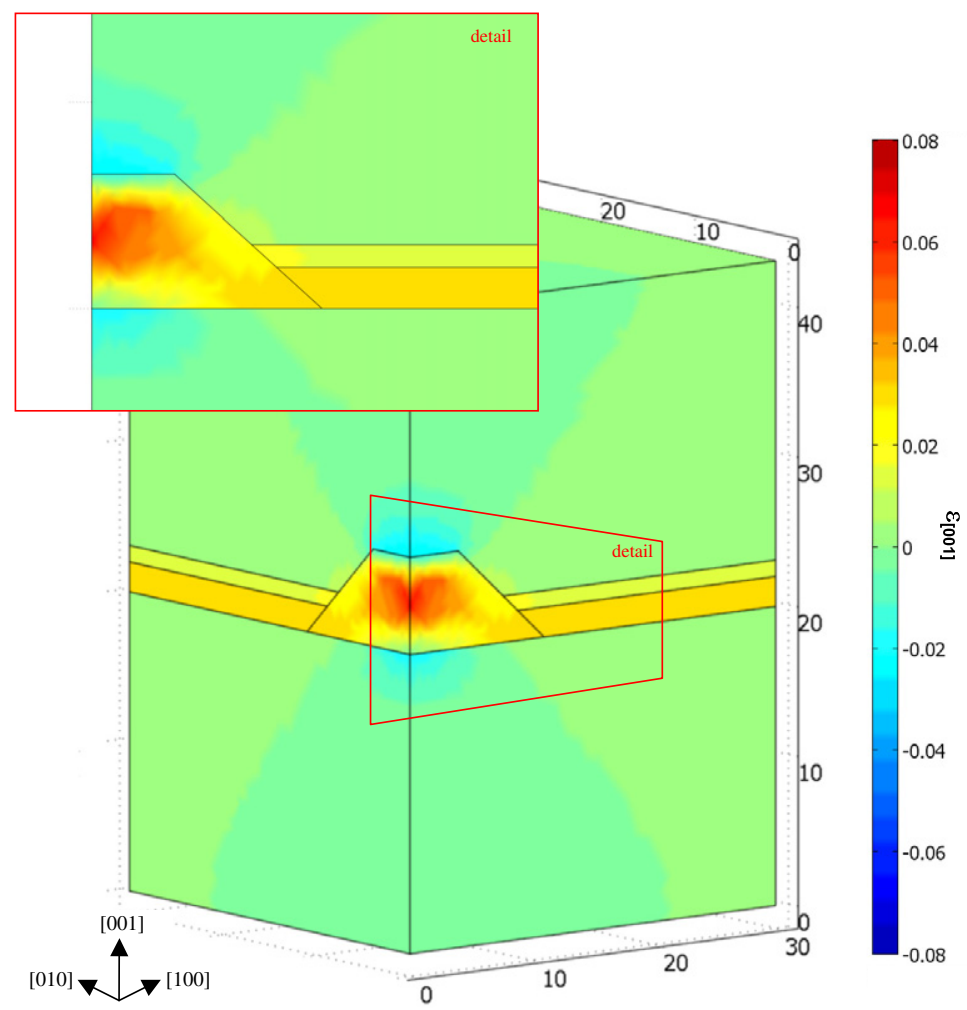

Figure 6. $\varepsilon_{[001]}$ strain distribution of the InAs QD simulated by the finite element method. A detail of this strain component in the middle plane of the QD is shown. 
Table 1. Basic parameters at $300 \mathrm{~K}$; mechanical properties of semiconductor compounds referred to in this work used for FEM simulations

\begin{tabular}{llllll}
\hline Property & Symbol & Units & $\mathrm{Al}_{0.15} \mathrm{Ga}_{0.85} \mathrm{As}$ & $\mathrm{GaAs}$ & $\mathrm{InAs}$ \\
\hline Lattice constant & $a$ & $\AA$ & 5.65447 & 5.65325 & 6.05830 \\
Young's modulus & $E$ & $\mathrm{~Pa}$ & $8.503 \times 10^{10}$ & $8.59 \times 10^{10}$ & $5.14 \times 10^{10}$ \\
Poisson ratio & $v$ & - & 0.325 & 0.31 & 0.35 \\
Elastic constants & $c_{11}$ & $\mathrm{~Pa}$ & $1.1901 \times 10^{11}$ & $1.19 \times 10^{11}$ & $8.34 \times 10^{10}$ \\
& $c_{12}$ & $\mathrm{~Pa}$ & $5.428 \times 10^{10}$ & $5.34 \times 10^{10}$ & $4.54 \times 10^{10}$ \\
& $c_{44}$ & $\mathrm{~Pa}$ & $5.9325 \times 10^{10}$ & $5.96 \times 10^{10}$ & $3.95 \times 10^{10}$ \\
\hline
\end{tabular}

are predominantly tetrahedral and a finer mesh is developed in areas where strain changes are expected.

\subsection{Elastic constants}

Anisotropic behaviour and values at $300 \mathrm{~K}$ are assumed for elastic constants. This last approximation has been shown to be reasonable for semiconducting materials in several works $[34,35]$. Properties of InAs, GaAs and $\mathrm{Al}_{0.15} \mathrm{Ga}_{0.85} \mathrm{As}$ have been taken from [36] and are given in table 1.

Vegard's law is assumed for the determination of $\mathrm{Al}_{0.15} \mathrm{Ga}_{0.85} \mathrm{As}$ and $\operatorname{In}_{x} \mathrm{Ga}_{(1-x)}$ As elastic constants and reticular coefficient. As an initial condition, a strain $\varepsilon_{0}$ corresponding to the reticular misfit of each layer with respect to the confining layer $\left(\mathrm{Al}_{0.15} \mathrm{Ga}_{0.85} \mathrm{As}\right)$ is introduced in subdomains (2)-(4) given by equation (1). Due to the non-uniformity in the composition, the initial strain changes with the position, $\vec{r}$ [37]. Then, the system evolves to an equilibrium state.

$$
\varepsilon_{0}(\vec{r})=\frac{a_{\mathrm{In}_{y} \mathrm{Ga}_{(1-y)} \mathrm{As}}(\vec{r})-a_{\mathrm{Al}_{0.15} \mathrm{Ga}_{0.85} \mathrm{As}}}{a_{\mathrm{Al}_{0.15} \mathrm{Ga}_{0.85} \mathrm{As}}} .
$$

Figure 6 shows the results of the FEM simulation. Maximum and minimum $\varepsilon_{[001]}$ strain values with respect to the unstrained $\mathrm{Al}_{0.15} \mathrm{Ga}_{0.85}$ As lattice are 0.064 and -0.027 respectively. Maximum strain is located in the core of the QD, owing to the higher In composition. On the other hand, minimum strain is observed on top of the dot. As compared to that of WL, that grows pseudomorphically on the LCL, the QD strain can relax parallel to the heterointerfaces. Hence, the AlGaAs UCL that grows on top of the QD undergoes a tensile strain that results in the negative value of $\varepsilon_{[001]}$.

The FEM model also enables us to find preferential sites for stacking fault nucleation. In this way, the 3D model was divided into slices in [001] for a better visualization of the strain. Then, a half plane in the wetting layer was found containing a profile, from the inner QD to the outside, where strain abruptly increases and then relaxes, as depicted in figure 7. The most strained region in this plane of the model corresponds to the place where stacking faults frequently appear in the studied samples.

Quantitative comparison of both determined and calculated strain is limited by (i) the unmeasured effect of deformed planes in the 3D nanostructure to the situation of the local maxima in HRTEM images, (ii) the thin foil relaxation experimented in the TEM sample, (iii) the use of bulk properties and the continuous matter approach in FEM calculations and (iv) the difference between the projected strain in the HRTEM image and the calculated one at a certain plane in the FEM model. Nevertheless, a qualitative comparison is possible, making unambiguous the existence of a compressive strain over the QD and the maximum strain in its core.

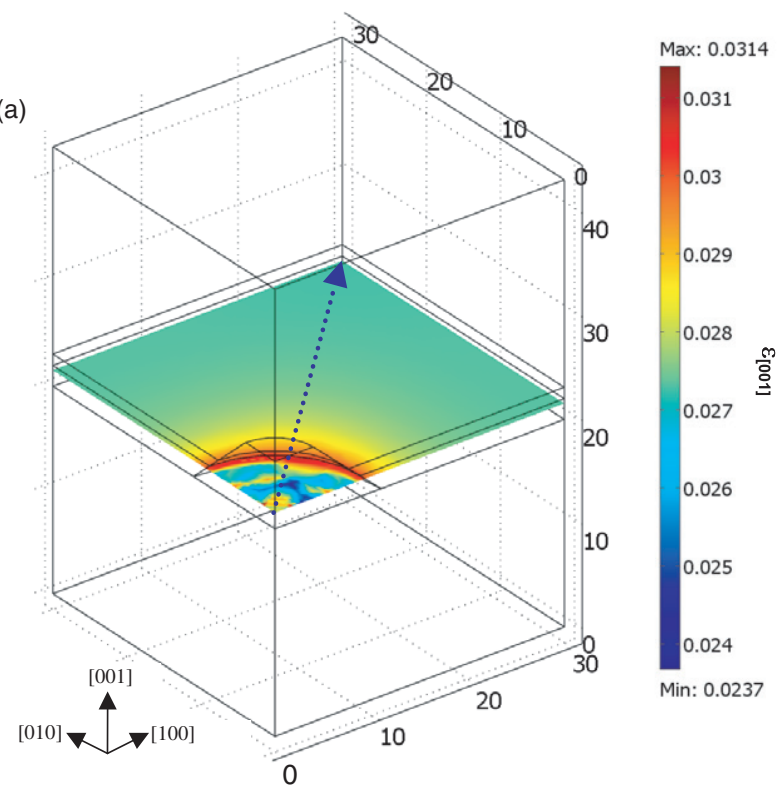

(b)

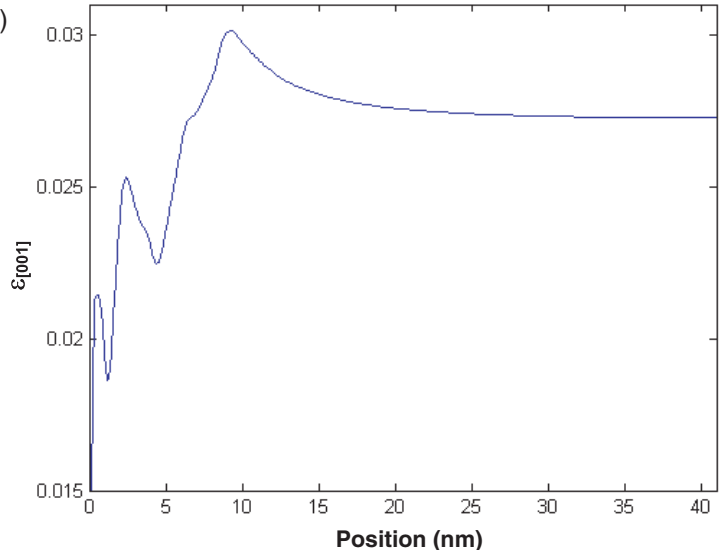

Figure 7. (a) $\varepsilon_{[001]}$ strain in a (001) plane inside the wetting layer. The highest strain values are found in the region where the wetting layer joins the QD. (b) $\varepsilon_{[001]}$ strain profile corresponding to the dotted blue line in (a).

\section{Conclusions}

Strain distribution of In(Ga)As/AlGaAs self-assembled semiconductor quantum dots (QDs) has been analysed by two different approaches. First, the Peak Pairs method has been used to map the strain from HRTEM micrographs, showing

- a maximum strain value in the growth direction of $0.058 \pm$ 0.015 , placed in the inner part of the QD, 
- a negative strain region over the QD due to the change of the on-plane (001) lattice parameter caused by the elastic relaxation of the QD, and

- a clear localization of the QD, delimiting its size and shape.

On the other hand, strain distribution has been obtained with FEM elasticity theory simulation on a 3D QD model. This simulation depicts

- a maximum strain value in the growth direction of 0.064 , placed in the inner part of the QD,

- a minimum negative strain in the region of the upper AlGaAs layer over the QD

- a high strain concentration area in the area of the wetting layer that surrounds the QD, where stacking faults are frequently observed.

The agreement between both calculated and simulated strain maps reveals that these techniques can be used to get consistent strain information on nanostructured materials as a qualitative approach. The correct application of both methods allows the localization of critical strain zones in semiconductor quantum dots, predicting the nucleation of defects, and being a very useful tool for the design of semiconductor devices.

\section{Acknowledgments}

This work was supported by Spanish MEC NANOSELF II project (TEC2005-05781-C03-02/MIC), the SANDiE Network of Excellence (contract No NMP4-CT-2004-500101) and the Junta de Andalucía (PAI research groups TEP-0120 and TIC-145; project PAI05-TEP-00383). TEM measurements were carried out in the DME-SCCYT-UCA.

\section{References}

[1] Stranski I N and Krastanow L 1937 Sitz.ber. Akad. Wiss. Wien, Math.-Nat.wiss. Kl. 146797

[2] Arakawa Y and Sakaki H 1982 Appl. Phys. Lett. 40 939-41

[3] Chen S D, Chen Y Y and Lee S C 2005 Appl. Phys. Lett. 86253104

[4] Tang S F, Chiang C D, Weng P K, Gau Y T, Luo J J, Yang S T, Shih C C, Lin S Y and Lee S C 2006 IEEE Photon. Technol. Lett. 18 986-8

[5] Li L H, Rossetti M, Fiore A and Patriarche G 2006 Electron. Lett. 42 638-40

[6] Karachinsky L Y et al 2005 Electron. Lett. 41 478-80

[7] Lundstrom T, Schoenfeld W, Lee H and Petroff P M 1999 Science 286 2312-4

[8] Kroutvar M, Ducommun Y, Finley J J, Bichler M, Abstreiter G and Zrenner A 2003 Appl. Phys. Lett. 83 443-5

[9] Maximov M V et al 2000 Phys. Rev. B 62 16671-80
[10] Kuo M K, Lin T R, Liao B T and Yu C H 2005 Physica E 26 199-202

[11] Davies J H 1998 J. Appl. Phys. 84 1358-65

[12] Jin-Phillipp N Y and Phillipp F 1999 J. Microsc. 194 161-70

[13] Nabetani Y, Yamamoto N, Tokuda D and Sasaki A 1995 J. Cryst. Growth 146 363-7

[14] Sears K, Wong-Leung J, Tan H H and Jagadish C 2006 J. Appl. Phys. 99113503

[15] Maksimov M V et al 2004 Semiconductors 38 1207-11

[16] Shamirzaev T S, Gilinsky A M, Kalagin A K, Toropov A I, Gutakovskii A K and Zhuravlev K S 2006 Semicond. Sci. Technol. 21 527-31

[17] Rosenauer A, Kaiser S, Reisinger T, Gebhardt W and Gerthsen D 1996 Optik 102 63-9

[18] Hÿtch M J, Snoeck E and Kilaas R 1998 Ultramicroscopy 74 131-46

[19] Galindo P L, Kret S, Sanchez A M, Laval J Y, Yáñez A, Pizarro J, Guerrero E, Ben T and Molina S I 2007 Ultramicroscopy 107 1186-93

Galindo P L, Yáñez A, Pizarro J, Guerrero E, Ben T and Molina S I 2005 Microscopy of Semiconducting Materials (Oxford) ed A G Cullis (Bristol, UK: Springer) pp 191-4

[20] Liu G R and Quek Jerry S S 2002 Semicond. Sci. Technol. $17630-43$

[21] Franchi S, Trevisi G, Seravalli L and Frigeri P 2003 Prog. Cryst. Growth Charact. Mater. 47 166-95

[22] Briones F and Ruiz A 1991 J. Cryst. Growth 111 194-9

[23] Bosacchi A, Frigeri P, Franchi S, Allegri P and Avanzini V 1997 J. Cryst. Growth 175/176 771-6

[24] Gurioli M, Testa S, Altieri P, Sanguinetti S, Grilli E, Guzzi M Trevisi G, Frigeri P and Franchi S 2003 Physica E 17 19-21

[25] Heyn Ch 2001 Phys. Rev. B 64165306

[26] Muraki K, Fakatsu S and Shiraki Y 1992 Appl. Phys. Lett. 61 557-9

[27] Rosenauer A, Gerthsen D, Van Dyck D, Arzberger M, Böhm G and Abstreiter G 2001 Phys. Rev. B 64245334

[28] Offermans P, Koenraad P M, Nötzel R and Wolter J H 2005 Appl. Phys. Lett. 87111903

[29] Zhi D, Davock H, Murray R, Roberts C, Jones T S, Pashley D W, Goodhew P J and Joyce B A $2001 \mathrm{~J}$. Appl. Phys. 89 2079-83

[30] Walther T, Cullis A G, Norris D J and Hopkinson M 2001 Phys. Rev. Lett. 86 2381-4

[31] Wang P, Bleloch A L, Falke M, Goodhew P J, Ng J and Missous M 2006 Appl. Phys. Lett. 89072111

[32] Fleming R M, McWhan D B, Gossard A C, Wiegmann W and Logan R A 1980 J. Appl. Phys. 51 357-63

[33] Lee D T and Schachter B 1980 Int. J. Comput. Inf. Sci. 9 219-242

[34] Andreev A D, Downes J R, Faux D A and O'Reilly E P 1999 J. Appl. Phys. 86 297-305

[35] Faux D A and Haigh J 1990 J. Phys.: Condens. Matter 210289

[36] Levinstein M, Rumyantsev S and Shur M (ed) 1996 Handbook Series on Semiconductor Parameters vol 1 (London: World Scientific)

Levinstein M, Rumyantsev S and Shur M (ed) 1999 Handbook Series on Semiconductor Parameters vol 2 (London: World Scientific)

[37] Chu H J and Wang J 2005 J. Appl. Phys. 98034315 